\title{
Auricular Acupuncture Treatment for Insomnia: A Systematic Review
}

\author{
HAI YONG CHEN, B.Sc., ${ }^{1,3, *}$ YAN SHI, Ph.D., ${ }^{1, *}$ CHI SUN NG, B.Sc., ${ }^{1}$ SAI MAN CHAN, B.Sc., ${ }^{1}$ \\ KEN KIN LAM YUNG, Ph.D., ${ }^{2}$ and QING LING ZHANG, Ph.D. ${ }^{1}$
}

\begin{abstract}
Objectives: To review trials on the efficacy and safety of auricular acupuncture (AA) treatment for insomnia and to identify the most commonly used auricular acupoints for treating insomnia in the studies via a frequency analysis.

Data sources: The international electronic databases searched included: (1) AMED; (2) the Cochrane library; (3) CINAHL; (4) EMBASE; and (5) MEDLINE. ${ }^{\circledR}$ Chinese electronic databases searched included: (1) VIP Information; (2) CBMdisc; and (3) CNKI.

Study selection: Any randomized controlled trials using AA as an intervention without using any co-interventions for insomnia were included. Studies using AA versus no treatment, placebo, sham AA, or Western medicine were included.

Data extraction: Two (2) independent reviewers were responsible for data extraction and assessment. The efficacy of AA was estimated by the relative risk (RR) using a meta-analysis.

Results: Eight hundred and seventy eight (878) papers were searched. Six (6) trials (402 treated with AA among 673 participants) that met the inclusion criteria were retrieved. A meta-analysis showed that AA was chosen with a higher priority among the treatment subjects than among the controls $(p<0.05)$. The recovery and improvement rates produced by AA was significantly higher than those of diazepam $(p<0.05)$. The rate of success was higher when AA was used for enhancement of sleeping hours up to 6 hours in treatment subjects $(p<0.05)$. The efficacy of using Semen vaccariae ear seeds was better than that of the controls $(p<$ $0.01)$; while magnetic pearls did not show statistical significance $(p=0.28)$. Six $(6)$ commonly used auricular acupoints were Shenmen (100\%), Heart (83.33\%), Occiput (66.67\%), Subcortex (50\%), Brain and Kidney (each $33.33 \%$, respectively).

Conclusions: AA appears to be effective for treating insomnia. Because the trials were low quality, further clinical trials with higher design quality, longer duration of treatment, and longer follow-up should be conducted.

\section{INTRODUCTION}

$\mathbf{I}_{\mathrm{i}}^{\mathrm{n}}$ nsomnia is a common condition that includes difficulty in initiating sleep, maintaining sleep, waking up too early, and nonrestorative or poor-quality sleep, all of which are commonly associated with symptoms of daytime functional impairment. ${ }^{1}$ According to a World Health Organization

(WHO) collaborative study in 14 countries, $16 \%$ of correspondents had difficulty falling asleep. Twenty-five percent $(25 \%)$ of the correspondents either experienced difficulty staying asleep or woke up too early. ${ }^{2}$ The Sleep in America Poll, conducted by the National Sleep Foundation in the United States, reported that $54 \%$ of correspondents experienced at least one symptom of insomnia a few nights per
\end{abstract}

\footnotetext{
${ }^{1}$ School of Chinese Medicine, and ${ }^{2}$ Department of Biology, Hong Kong Baptist University, Hong Kong.

${ }^{3}$ School of Chinese Medicine, University of Hong Kong, Hong Kong.

*These authors contributed equally to this paper.
} 
week within the year prior to the poll. One third (33\%) experienced at least one symptom every night or almost every night. ${ }^{3}$ The estimated prevalence of insomnia with daytime consequences was 9\%-15\%. ${ }^{4}$ Daytime consequences and functional impairment included daytime sleepiness, irritability, depression, and social and occupational role impairment, et cetera, which caused a heavy economic burden to the society. ${ }^{5}$ Chilcott et al. ${ }^{6}$ estimated that the total annual cost of insomnia was approximately $\$ 30-\$ 35$ billion in the United States.

Acupuncture has been widely used to treat various diseases and complaints for thousands of years in China. This treatment is considered to be an inexpensive and effective therapy with few adverse effects. Worldwide acceptance of the therapy is steadily increasing. In 1994, approximately 1 million people annually were using acupuncture as an alternative treatment in the United States. ${ }^{7}$ One type of the acupuncture, auricular acupuncture (AA), was first described in the $1950 \mathrm{~s}^{8}$ It involves stimulations at specific acupoints on the outer ear. Abbate ${ }^{9}$ summarized 15 modalities used in AA, such as needles, pellets (Semen vaccariae ear seeds), magnetic pearls, electricity, et cetera. Current studies show that AA helps relieve a large number of diseases and complaints (i.e., anxiety, ${ }^{10}$ postoperative pain,,${ }^{11,12}$ chronic low-back pain, ${ }^{13}$ and problems related to spinal-cord injuries ${ }^{14}$ ).

Many clinical studies support AA as an effective therapy for insomnia. After an extensive search in the Cochrane library, in major international medical databases, and in Chinese medical databases, we found there was much recent advancement in this area of study. To provide clinicians with updated high-quality evidence supporting the use of AA in treating insomnia, we carried out a systematic review of AA studies. The objective of this study was to investigate the efficacy and safety of AA treatment for insomnia in studies and to identify the most commonly used auricular acupoints for treating insomnia by a frequency analysis.

\section{METHODS}

\section{Search strategy}

All the databases were searched from their starting date to April 2006. The international electronic databases searched were: (1) AMED; (2) the Cochrane library; (3) CINAHL; (4) EMBASE; and (5) MEDLINE. ${ }^{\circledR}$ The Chinese electronic databases searched included: (1) VIP Information; (2) CBMdisc; and (3) CNKI. Key words used in the search included: auricular, oto\$, acupuncture, acupressure, acupoint, insom\$, sleep\$, wakeful\$ (“\$” indicates that this term has been truncated). ${ }^{15}$ The search strategy with combined key words included: (auricular OR oto\$) AND (acupuncture OR acupressure OR acupoint) AND (insom\$ OR sleep\$ OR wakeful\$). "AND" indicates retrieved results including all the search terms; "OR" indicates retrieved results including at least one of the search terms.

The results of the electronic search, bibliographic searches, and hand searches were reviewed by 2 independent reviewers (H.Y. Chen and C.S. Ng). The paper titles and abstracts were retrieved and assessed for their potential relevance. Then, the full text of related papers were obtained and reviewed independently by 2 reviewers, based on the inclusion and exclusion criteria of the studies. The reviewers were not blinded to the names of the authors, institutions, or the journal of publication of each study. After selection of studies, the data from the included studies were extracted. A standardized data extraction form was used. The data were extracted by the first reviewer (H.Y. Chen) and verified by the second reviewer (C.S. Ng). Any discrepancies were corrected by referring to the original papers. The extracted data included population characteristics, basic demographic data, diagnostic criteria, treatment duration and method, treatment effect measurement, incidence of adverse effects, and quality of study design.

\section{Selection criteria}

Only randomized controlled trials (RCTs) and papers written in the Chinese or English languages from the above databases were included. Quasirandomized trials and case studies were excluded.

Only those studies that used AA as the sole intervention were selected, while those that used AA as a co-intervention were excluded. Conversely, studies not using AA as an intervention were excluded. Only studies using no treatment, sham AA, placebo, or Western Medicine as control interventions were included. Selection criteria were not based on any special restrictions on the use of needles and other TCM manipulations (finger message, placing medical tape, etc.). Furthermore, studies were selected with no special restrictions on the use of auricular acupoints, needles, other manipulation methods, and duration of studies. In addition, studies comparing different groups of auricular acupoints, different times for receiving treatments, and different ways for stimulating the auricular acupoints were excluded.

\section{Quality assessment of the trials}

Two independent reviewers assessed the quality of each trial according to four predetermined criteria: (1) randomization; (2) allocation concealment; (3) blinding; and (4) withdrawals and dropouts. Each item of 1 trial was classified into four grades: (1) adequate; (2) inadequate; (3) unclear; or (4) not used according to the Cochrane reviewers' handbook. ${ }^{16}$ Based on those four criteria, the quality of studies were classified into three grades: ${ }^{17}$ (1) Low risk of bias for studies, with adequate grade for all items; (2) moderate risk of bias for studies, with one or more inadequate grades; and (3) high risk of bias for studies, with one or more unclear. 
These trials were also assessed via the Jadad scale. ${ }^{18}$ The Jadad scale was used for quality assessment of the included papers. It addressed 3 major aspects of research study quality: (1) randomization;(2) blinding; and (3) withdrawal. The scale consisted of 5 questions:

1. Was the study randomized?

2. Was the randomization adequately described?

3. Was the study double-blinded?

4. Was the blindedness described adequately?

5. Was there a description of withdrawals? One (1) point was assigned for each question that received a "yes" answer. Research studies that were assigned a total of 1-2 points were regarded as low quality, while trials with a total of 3 points or more were considered to be high quality.

\section{Data analysis}

Review Manager Software 4.2 (www.cc-ims.net/RevMan) was used for data analysis. A Chi-square test was performed to assess the heterogeneity of studies. The random-effect model was chosen because this model offers a wider, more conservative confidence interval (CI). The data were dichotomized and expressed as a relative ratio (RR) with a $95 \%$ CI.

\section{RESULTS}

\section{Description of the trials}

In this review, 878 research papers were searched. Only 6 randomized controlled trials were included. Five (5) ${ }^{19-23}$ were in Chinese, while $1^{24}$ was in English. The 6 studies were all conducted in mainland China or Hong Kong. A total of 673 patients were included in the 6 studies, ${ }^{18-23}$ in which 402 patients received AA treatment. Four (4) of the trials ${ }^{19,21-23}$ used Western medication for the control groups; 1 trial $^{22}$ used only routine unit care, and another ${ }^{24}$ used sham AA. Only $1^{24}$ trial reported follow-up events (Table 1).

\section{Quality assessment}

Methodological quality. Among the 6 studies that were included, all authors claimed that participants were randomly divided into treatment and control groups. None of the included studies described how the participants were randomized. In addition, none of them mentioned anything regarding allocation concealment. Zhang et al. ${ }^{20}$ reported having adopted diagnostic criteria published in Traditional Chinese Medicine Diagnosis ${ }^{25}$ and Wang et al. ${ }^{23}$ reported adopting the diagnostic criteria published in Diagnostic and Curative Criteria: TCM Internal Medicine. ${ }^{26}$ Four (4) studies ${ }^{19,21,22,24}$ did not mention any Western insomnia diagnosis. Only two of the trials listed the exclusion criteria for trial subjects, ${ }^{23,24}$ while in the other trials, ${ }^{19-22}$ the exclusion criteria for study subjects were not listed. According to the Cochrane reviewers' handbook, all studies were at a high risk of bias. According to the Jadad scale, the overall quality of all the included studies was low. Among the 6 included trials, 5 trials received a score of 1 , and 1 trial received a score of 2 . No trial scored a 3 or above (high quality) (Table 2).

Efficacy assessment. Four (4) trials ${ }^{19-22}$ were based on sleep time and reduction of insomnia (self-setting measurement). One (1) trial ${ }^{23}$ adopted the effectiveness scale published in the Diagnostic and Curative Criteria: TCM Internal Medicine, ${ }^{26}$ which involves clinic symptom and emotion changes. Another trial ${ }^{24}$ used wrist actigraphy recording the activity of participants during sleep and monitored sleep behaviors.

For subgroup analysis, 5 trials $^{19,21,23,24}$ expressed effectiveness by using total recovery and improvement rates. The effectiveness of AA was better than that of the control interventions, with statistical significance. The relative risk (RR), in the random-effect model, was 1.93 (95\% CI, $1.40-2.66 ; p<0.05$ ) (Fig. 1, subcategory 01 ).

Two (2) trials ${ }^{19,20}$ mentioned sleep time as an effectiveness scale. The treatment was considered as effective by increasing nocturnal sleeping hours up to at least 6 hours, with good maintenance of sleep and feeling refreshed upon awakening. RR was 2.64 (95\% CI, 1.22-5.72; $p<0.05$ ). Heterogeneity was present $(p=0.05)$. This result suggested that AA treatment was favored over the control interventions for treating insomnia (Fig. 1, subcategory 02).

Three (3) trials ${ }^{19,20,22}$ used $S$. vaccariae ear seeds for AA, which assessed the rates of recovery and improvement at the same time. The treatment groups showed more efficacy than the control ( $\mathrm{RR}=1.80 ; 95 \% \mathrm{CI}, 1.42-2.28 ; p<0.05)$. The heterogeneity of $S$. vaccariae ear seeds was present $(p=$ $0.32)$. S. vaccariae ear seeds were statistically favored over the controls $(p<0.01)$, while the magnetic pearls were not statistically favored $(\mathrm{RR}=2.09 ; 95 \% \mathrm{CI}, 0.54-8.07 ; p=$ 0.28 ; Fig. 2). Two (2) trials ${ }^{20,23}$ used diazepam, a commonly used drug to relieve insomnia, as a control intervention. The rates of recovery and improvement produced by AA were more favored than that of diazepam, with statistical significance. The RR was statistically significant with a value of 1.41 (95\% CI 1.12-1.77; $p<0.05$ ). Heterogeneity was present $(p=0.27$; Fig. 3$)$.

Adverse effects and withdrawals. None of the included studies mentioned anything regarding the adverse effects of receiving AA treatment. Only one (1) trial ${ }^{24}$ reported the number of and reasons for withdrawalof participants in the study.

\section{Commonly used auricular acupoints}

Twelve (12) main auricular acupoints for treating insomnia were reported in the 6 trials. Those auricular acupoints were Shenmen (100\%), Heart (83.33\%), Occiput (66.67\%), 


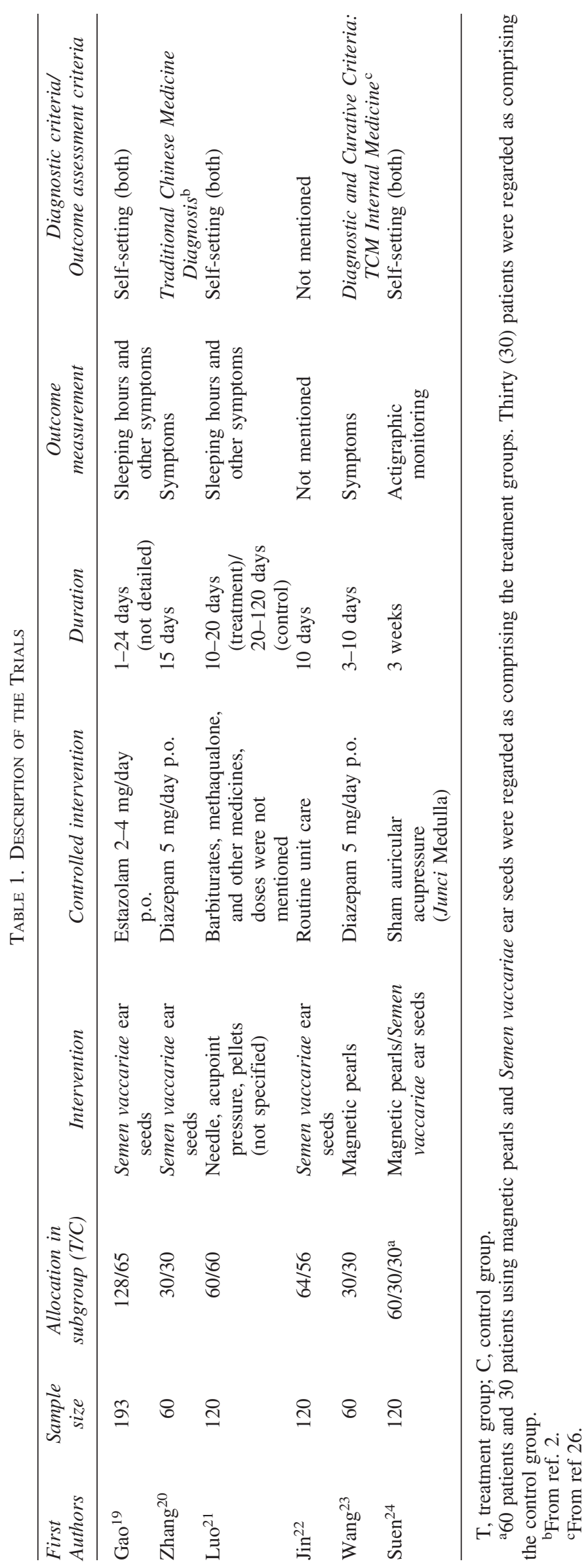


Table 2. Quality Evaluation of Included Studies

\begin{tabular}{|c|c|c|c|c|c|c|c|c|}
\hline \multirow[b]{2}{*}{ Study } & \multirow[b]{2}{*}{$\begin{array}{c}\text { Treatment } \\
\mathrm{N}\end{array}$} & \multirow[b]{2}{*}{$\begin{array}{c}\text { Control } \\
\mathrm{N}\end{array}$} & \multicolumn{5}{|c|}{ Quality assessment } & \multirow[b]{2}{*}{$\begin{array}{c}\text { Jadad scale } \\
\text { score }\end{array}$} \\
\hline & & & Ran. & $\begin{array}{l}\text { All. } \\
\text { Con. }\end{array}$ & Blind. & $\begin{array}{l}\text { With./ } \\
\text { Drop. }\end{array}$ & $\begin{array}{l}\text { Total } \\
\text { Score }\end{array}$ & \\
\hline $\mathrm{Gao}^{19}$ & 128 & 65 & B & $\mathrm{C}$ & C & $\mathrm{C}$ & H.R.B. & 1 \\
\hline Zhang $^{20}$ & 30 & 30 & $\mathrm{~B}$ & $\mathrm{C}$ & $\mathrm{C}$ & $\mathrm{C}$ & H.R.B. & 1 \\
\hline $\mathrm{LuO}^{21}$ & 60 & 60 & $\mathrm{~B}$ & $\mathrm{C}$ & $\mathrm{C}$ & $\mathrm{C}$ & H.R.B. & 1 \\
\hline $\mathrm{Jin}^{22}$ & 64 & 56 & $\mathrm{~B}$ & $\mathrm{C}$ & $\mathrm{C}$ & $\mathrm{C}$ & H.R.B. & 1 \\
\hline Wang 23 & 30 & 30 & $\mathrm{~B}$ & $\mathrm{C}$ & $\mathrm{C}$ & $\mathrm{C}$ & H.R.B. & 1 \\
\hline Suen $^{24}$ & 90 & 30 & $\mathrm{~B}$ & $\mathrm{C}$ & $\mathrm{C}$ & $\mathrm{C}$ & H.R.B. & 2 \\
\hline Subtotal & 402 & 271 & & & & & & \\
\hline Total & 673 & & & & & & & \\
\hline
\end{tabular}

Ran., randomization; All. Con., allocation concealment; Blind., Blindedness; With./drop, withdrawal/ dropout.

Assessments: $\mathrm{B}=$ inadequate; $\mathrm{C}=$ unclear; H.R.B. = high risk of bias.

Subcortex (50\%), Brain and Kidney (33.33\%), Liver, Spleen, Anterior ear lobe, Forehead, Sympathetic nerve, and Endocine (all 16.67\%, respectively) (Table 3).

\section{DISCUSSION}

\section{Quality assessment of included trials}

Randomization. Randomization is a statistical method used to protect experiments from the influences of factors that are known to affect outcomes, and from unidentified factors as well. Via randomization, the baseline characteristics in each of the randomized groups are thoroughly reviewed and equal distribution of demographic characteristics and morbidity characteristics is ensured. The homogeneity of the control and treatment groups with respect to all other characteristics, traits, and measures is ensured.
Therefore, randomization is a very important process in eliminating bias in experiments.

However, the included trials did not seem to place enough emphasis on this important process. Among the 6 trials, all investigators claimed to perform randomization, but none of them reported the methods used. It is questionable whether randomization was properly performed, with regard to the large number of excluded clinical trials, which claimed to perform randomization, but were using the wrong methods.

Blinding. Blinding is a statistical method used to increase the likelihood of patients having equivalent experiences during the course of a clinical trial. Although this is a very important process, none of the included trials used doubleblinding. This may partially be related to the difficulty in performing blinding in AA. Because it is a unique treatment method with a long history of practice, Chinese practitioners are familiar with the process and "feeling" of AA treatment. It would be impossible to blind patients and physi-

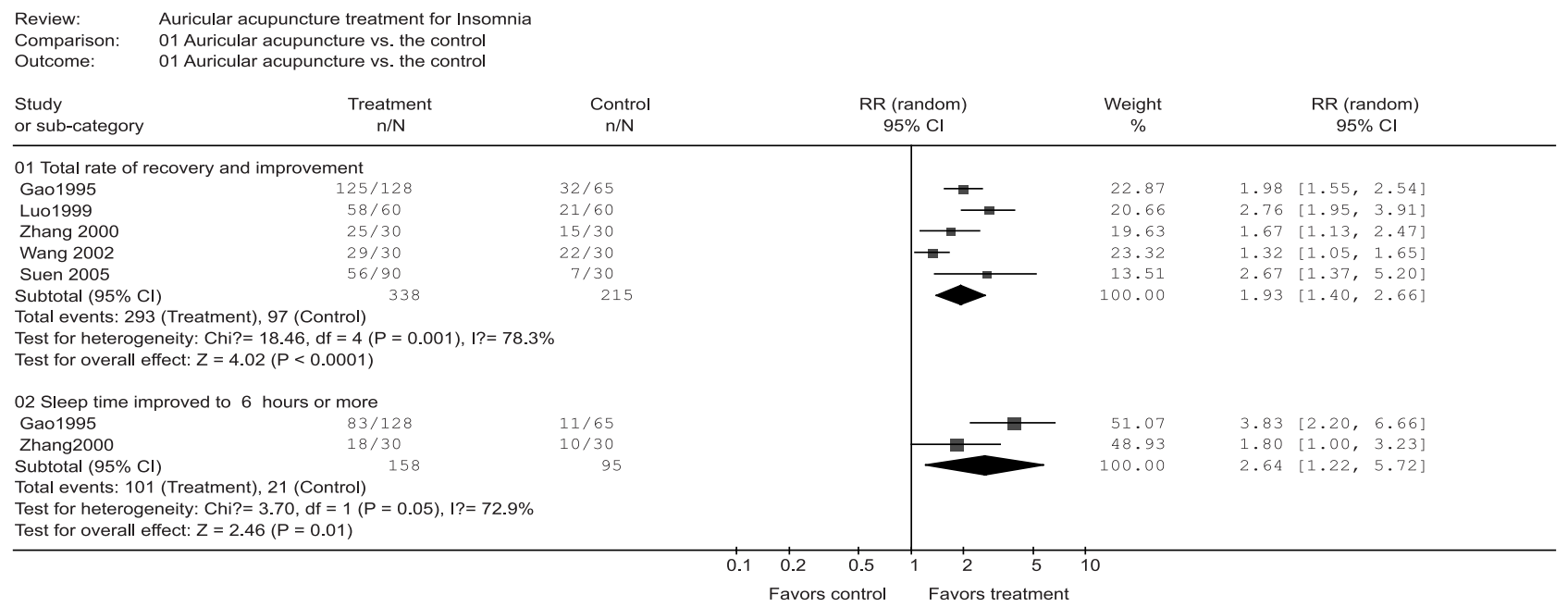

FIG. 1. Effectiveness analysis of treating insomnia with auricular acupuncture (treatment) compared to controls. 


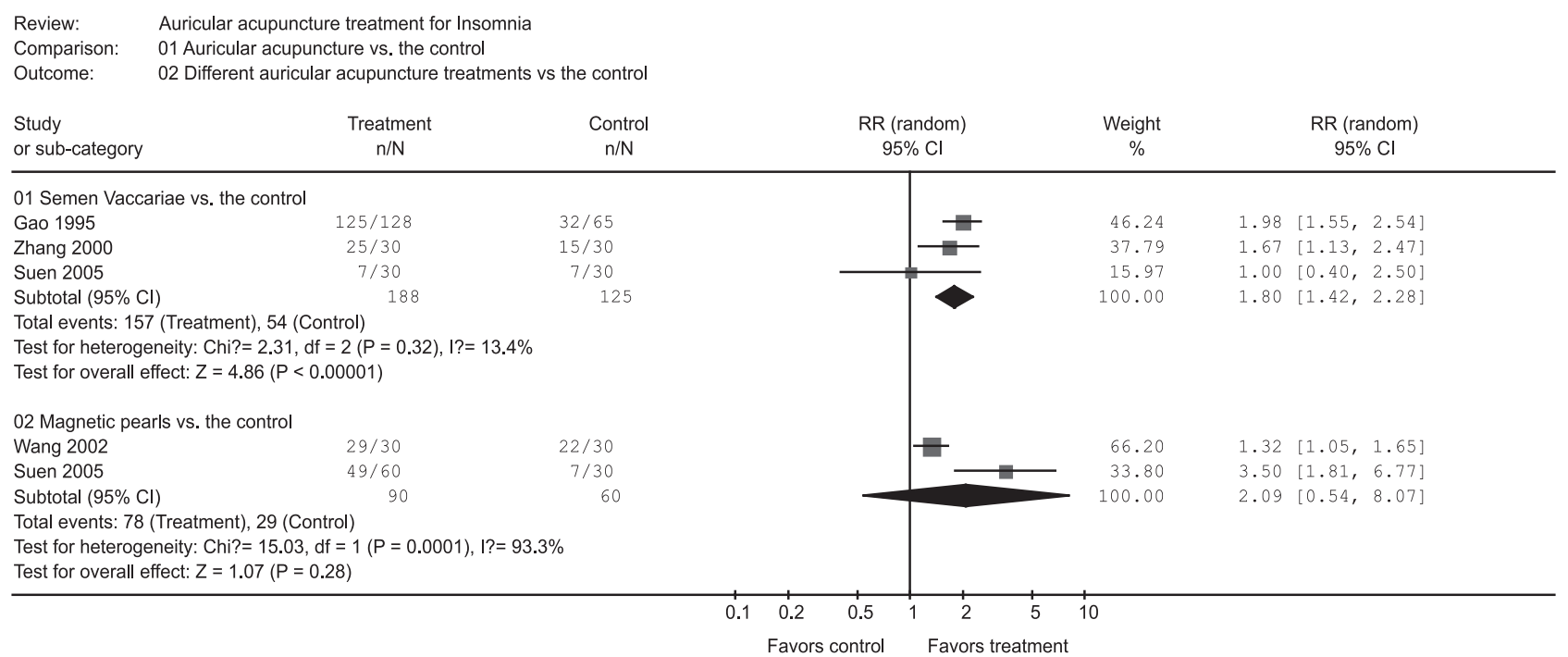

FIG. 2. Effectiveness analysis of treating insomnia with different auricular acupuncture treatments (Semen vaccariae ear seeds or magnetic pearls) compared to controls.

cians. The outcome assessor may be blinded, but the bias that the patients and physicians introduce cannot be removed or estimated. Further studies should be conducted on this aspect to provide the best method or substitute for blinding in AA trials.

\section{Research design}

Problems of inclusion, exclusion, and diagnostic criteria. Investigators of the included studies seemed to confuse the terms: "inclusion criteria," "exclusion criteria," and "diagnostic criteria." Most of these trials did not report the inclusion and exclusion criteria, and simply gave brief diagnostic criteria. It is suggested that inclusion and exclusion criteria should be stated, with diagnostic criteria placed as a subset of inclusion criteria.

Nonstandardized diagnostic criteria and effectiveness measurements. Only two (2) trials ${ }^{20,23}$ reported diagnostic criteria in the 6 included trials. Although these criteria overlap to a certain degree, the baseline randomization of the meta-analysis is dampened. Adopting international research diagnostic criteria in these studies can solve this problem. Currently, there is no "gold standard" for diagnosing insomnia, probably because of the lack of understanding of the etiology of insomnia. However, some people have already developed research diagnostic criteria for insomnia, which await validation.

For effectiveness measurement, different tools were used. The Insomnia Severity Index (ISI), the Morning Questionnaire, the Athens Insomnia Index (ASI), and the Pittsburgh Sleep Quality Index (PSQI) were used. The most popular choice was to use self-defined effectiveness criteria, which are not validated. To increase external validity, it is suggested that internationally recognized and validated effectiveness measurement should be adopted to provide better baseline randomization for future analyses.

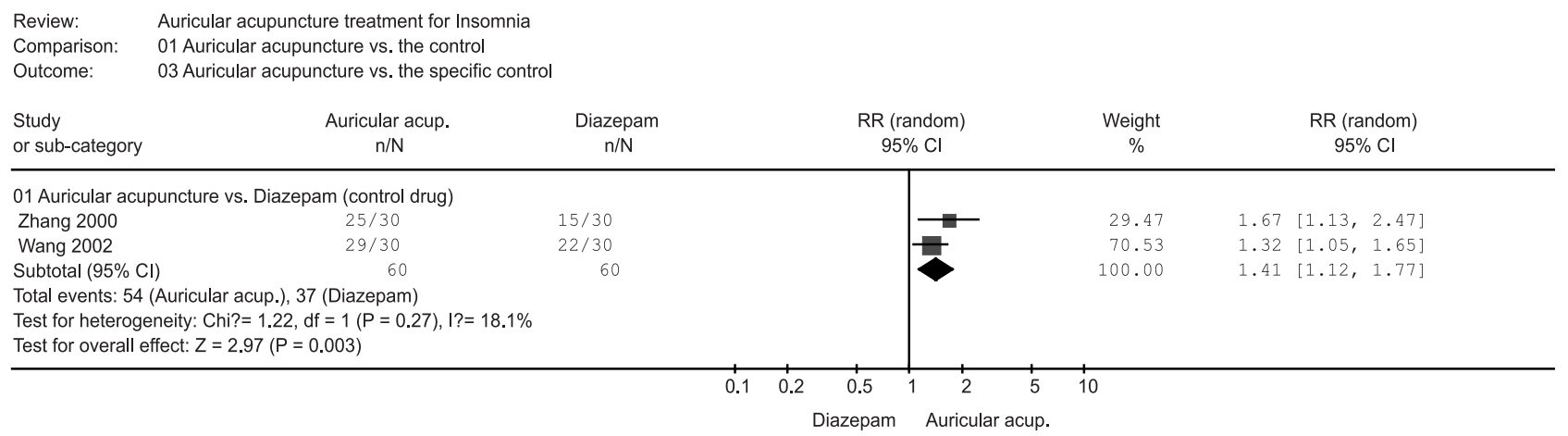

FIG 3. Effectiveness analysis of treating insomnia with auricular acupuncture compared to a specific control (Diazepam). Auricular acup., Auricular acupuncture. 
Table 3. Commonly Used Auricular Acupoints in Included Studies

\begin{tabular}{|c|c|c|c|c|c|c|c|c|}
\hline \multirow[b]{2}{*}{ Auricular acupoints } & \multicolumn{6}{|c|}{ Included studies } & \multicolumn{2}{|c|}{ Total } \\
\hline & $G a o^{19}$ & Zhang $^{20}$ & $\mathrm{Luo}^{21}$ & $\operatorname{Jin}^{22}$ & Wang 23 & Suen $^{24}$ & Frequency & $\%$ \\
\hline Shenmen & + & + & + & + & + & + & 6 & 100.0 \\
\hline Heart & + & + & + & - & + & + & 5 & 83.33 \\
\hline Occiput & - & + & + & + & - & + & 4 & 66.67 \\
\hline Subcortex & - & + & - & + & - & + & 3 & 50.00 \\
\hline Brain & + & - & + & - & - & - & 2 & 33.33 \\
\hline Kidney & - & - & + & - & - & + & 2 & 33.33 \\
\hline Forehead & - & + & - & - & - & - & 1 & 16.67 \\
\hline Sympathetic nerve & - & - & - & - & + & - & 1 & 16.67 \\
\hline Endocine & - & - & - & - & + & - & 1 & 16.67 \\
\hline Liver & - & - & - & - & - & + & 1 & 16.67 \\
\hline Spleen & - & - & - & - & - & + & 1 & 16.67 \\
\hline Anterior ear lobe & - & + & - & - & - & - & 16.67 & \\
\hline
\end{tabular}

+ , auricular acupoints mentioned in studies; -, auricular acupoints not mentioned in studies.

Unclear Western medicine dosages and names. In some of the included studies, investigators did not state clearly the dosage and name of Western drugs that were administered to patients. For example, Luo et al. ${ }^{21}$ simply stated that more than 3 tablets were given to the control group, without specifying the weight of each tablet or specific patients for different medicines. This introduced confusion about the drug dosages and led to difficulty in comparing the homogeneity of dosages used in different trials.

Absence of baseline measurements. For most of the trials, ${ }^{18,20-22}$ investigators only reported the baseline homogeneity for simple demographic data of participants (e.g., gender, age). Some of the authors ${ }^{20,24}$ did not report the baseline seriousness and duration of insomnia of the participants. It is questionable whether the participants in the control and treatment groups really had comparable degrees of illness.

Unclear duration of trials. The duration of trials was not clearly stated in some of the included studies. For example, $\mathrm{Gao}^{19}$ stated that the treatment and control groups had 10 days as a course. However, he did not mention how many courses were undertaken. Luo et al. ${ }^{21}$ mentioned that the treatment group received "treatment 1 or 2 days, 10 days as a course," but she did not specify the number of courses or real duration. This led to confusion in estimating the similarity of treatment durations between the studies.

Questions regarding adequacy of treatment. Among the 6 included studies, all trials failed to address the adequacy of treatment. Further assessment needs to be done to verify the adequacy of the treatment procedures because if treatment was not adequate, the conclusion of that treatment has efficacy is questionable.

Lack of follow-ups. Only 1 of the included trials stated the duration of follow-up clearly. ${ }^{24}$ This led to difficulty in accounting for the long-term efficacy of AA treatment for insomnia.

\section{Reporting of clinical trials}

Absence of withdrawal information. Only 1 of the 6 trials reported withdrawal information. ${ }^{24}$ In the absence of withdrawal reporting, information on handling of possible dropout data and the impact of dropouts on the reliability of trials is rendered suspect.

Absence of adverse-effect report. There is a common belief among Chinese people that Chinese medical treatments (e.g., herbal treatment, AA) have fewer side-effects compared to Western medication. Tempting as it might seem, there is no systematic research that verifies this claim. None of the included trials made any effort to report adverse effects that appeared during the treatments. The absence of adverse-effects reporting in these trials hindered the investigation regarding the safety of AA treatment for insomnia.

\section{CONCLUSIONS}

According to the meta-analysis, AA treatment of insomnia produces better rates of recovery and improvement than the control approaches. AA also produces better rates of recovery and improvement than diazepam for treating insomnia. AA treatment is favored over control interventions for increasing nocturnal sleeping hours up to 6 , with good sleep maintenance and feeling refreshed upon wakening. However, owing to the poor quality of included trials, it is suggested that RCTs with better methodology quality should be conducted in the future to find further endorsement for this conclusion.

Owing to the lack of information on follow-up, it is difficult to draw conclusions regarding the long-term efficacy 
of AA treatment for managing insomnia. In addition, nonreporting of adverse effects of using AA in these trials resulted in no conclusion regarding the safety of using AA treatment for patients with insomnia.

\section{ACKNOWLEDGMENTS}

We thank Dr. Neetu Usha Arora for critical reading of the manuscript. The research was supported by the Faculty Research Grant, Hong Kong Baptist University, Hong Kong.

\section{REFERENCES}

1. American Academy of Sleep Medicine. The International Classification of Sleep Disorders: Diagnostic and Coding Manual, 2nd ed. Westchester, IL: American Academy of Sleep Medicine; 2005.

2. Ustun T, Privett M, Lecrubier Y, et al. Form, frequency and burden of sleep problems in general health care: A report from the WHO Collaborative Study on Psychological Problems in General Health Care. European Psychiatry. 1996;11(suppl1): $5 \mathrm{~s}-10 \mathrm{~s}$.

3. National Sleep Foundation. Summary of Findings: 2005 Sleep in America Poll. Online document at: www.sleepfoundation. org/site/c.huIXKjM0IxF/b.2419039/k.14E4/2005_Sleep_in_ America_Poll.htm Accessed July 9, 2007.

4. Ohayon MM. Epidemiology of insomnia: What we know and what we still need to learn. Sleep Med Rev 2002;6:97-111.

5. Ozminkowski RJ, Wang S, Walsh JK. The direct and indirect costs of untreated insomnia in adults in the United States. Sleep 2007;30:263-273.

6. Chilcott LA, Shapiro CM. The socioeconomic impact of insomnia: An overview. Pharmacoeconomics 1996;10(suppl1): $1-14$.

7. Paramore LC. Use of alternative therapies: Estimates from the 1994 Robert Wood Johnson Foundation National Access to Care Survey. J Pain Symptom Manage 1997;13:83-89.

8. Wang SM, Peloquin C, Kain ZN. The use of auricular acupuncture to reduce preoperative anxiety. Anesthesia Analg 2001; 93:1178-1180.

9. Abbate S. Chinese Auricular Acupuncture. Boca Raton: CRC Press; 2004.

10. Wang SM, Maranets I, Weinberg ME, et al. Parental auricular acupuncture as an adjunct for parental presence during induction of anesthesia. Anesthesiology 2004;100:1399-1404.

11. Usichenko TI, Dinse M, Hermsen M, et al. Auricular acupuncture for pain relief after total hip arthroplasty—a randomized controlled study. Pain. 2005;114:320-327.

12. Usichenko TI, Kuchling S, Witstruck T, et al. Auricular acupuncture for pain relief after ambulatory knee surgery: A randomized trial. CMAJ Canadian Med Assoc J 2007;176: 179-183.

13. Sator-Katzenschlager SM, Scharbert G, Kozek-Langenecker $\mathrm{SA}$, et al. The short- and long-term benefit in chronic low back pain through adjuvant electrical versus manual auricular acupuncture. Anesthesia Analg 2004;98:1359-1364.

14. Wong AM, Leong CP, Su TY, et al. Clinical trial of acupuncture for patients with spinal cord injuries. Am J Phys Med Rehab 2003;82:21-27.

15. Cheuk DKL, Wong V. Acupuncture for insomnia (protocol). Cochrane Database Syst Rev 2005;3:CD005472.

16. Higgins J, Green S. Cochrane Handbook for Systematic Reviews of Interventions 4.2.6, Issue 4. Chichester, UK: John Wiley \& Sons, Ltd., 2006 [updated September 2006].

17. Bian Z, Wu T, Liu L, et al. Effectiveness of the Chinese herbal formula TongXieYaoFang for irritable bowel syndrome: A systematic review. J Altern Complement Med 2006;12:401407.

18. Jadad AR, Moore RA, Carroll D, et al. Assessing the quality of reports of randomized clinical trials: Is blinding necessary? Controlled Clinical Trials. 1996;17:1-12.

19. Gao Y. Insomnia treated by auricular acupressure in 128 cases. Shanghai J Acupunct Moxibustion. 1995;14:161-162.

20. Zhang J, Li J, Li YS, et al. A controlled study of treating insomnia by auricular acupressure and diazepam. Yunan Journal of Traditional Chinese Medicine 2000;21:37-38.

21. Luo ZP, Li XD, He YN, Han XY. Clinical observation of 367 cases of treating insomnia by oto-pressure. Heilongjiang J Trad Chinese Med 1993;1:45-48.

22. Jin RF. Stimulating auricular acupoint by Semen Vaccariae to treat internal diseases complicated with insomnia in 64 cases. Zhejiang J Trad Chinese Med 2003:213.

23. Wang ST. An observation on efficacy of auricular acupressure on insomnia using magnetic pearls. Anhui Clin J Trad Chinese Med 2002;14:165.

24. Suen LK, Wong TK, Leung AW. Effectiveness of auricular therapy on sleep promotion in the elderly. Am J Chinese Med 2002;30:429-449.

25. Zhu WF. Traditional Chinese Medicine Diagnosis. Shanghai: Shanghai Publishing House of Science and Technology, 1995.

26. The State Administration of Traditional Chinese Medicine of PRC. Diagnostic and Curative Criteria: TCM Internal Medicine (ZY 1001, 1-94), 1994.

Address reprint requests to: Hai Yong Chen, B.Sc.

School of Chinese Medicine University of Hong Kong 10 Sassoon Road Pokfulam Hong Kong

E-mail: hoiyong@gmail.com 


\section{This article has been cited by:}

1. Yang Li-Hua, Duan Pei-Bei, Du Shi-Zheng, Sun Jin-Fang, Mei Si-Juan, Wang Xiao-Qing, Zhang Yuan-Yuan. 2014. Efficacy of Auriculotherapy for Constipation in Adults: A Systematic Review and Meta-Analysis of Randomized Controlled Trials. The Journal of Alternative and Complementary Medicine 20:8, 590-605. [Abstract] [Full Text HTML] [Full Text PDF] [Full Text PDF with Links]

2. J. Vas, M. Modesto, I. Aguilar, C. d. S. Goncalo, F. Rivas-Ruiz. 2014. Efficacy and safety of auriculopressure for primary care patients with chronic non-specific spinal pain: a multicentre randomised controlled trial. Acupuncture in Medicine 32, 227-235. [CrossRef]

3. Crispian ScullyComplementary and alternative medicine 652-658. [CrossRef]

4. Prisco Michelle Kennedy, Jecmen Michael Cory, Bloeser Katharine J., McCarron Kelly K., Akhter Jeanette E., Duncan Alaine D., Balish Marshall S., Amdur Richard L., Reinhard Matthew J.. 2013. Group Auricular Acupuncture for PTSD-Related Insomnia in Veterans: A Randomized Trial. Medical Acupuncture 25:6, 407-422. [Abstract] [Full Text HTML] [Full Text PDF] [Full Text PDF with Links]

5. Chyi Lo, Wen-Chun Liao, Jen-Jiuan Liaw, Liang-Wen Hang, Jaung-Geng Lin. 2013. The Stimulation Effect of Auricular Magnetic Press Pellets on Older Female Adults with Sleep Disturbance Undergoing Polysomnographic Evaluation. Evidence-Based Complementary and Alternative Medicine 2013, 1-8. [CrossRef]

6. Guo-Shiou Liao, Maria Karmella Apaya, Lie-Fen Shyur. 2013. Herbal Medicine and Acupuncture for Breast Cancer Palliative Care and Adjuvant Therapy. Evidence-Based Complementary and Alternative Medicine 2013, 1-17. [CrossRef]

7. Regina Round, Gerhard Litscher, Frank Bahr. 2013. Auricular Acupuncture with Laser. Evidence-Based Complementary and Alternative Medicine 2013, 1-22. [CrossRef]

8. Wing-Fai Yeung, Ka-Fai Chung, Maggie Man-Ki Poon, Fiona Yan-Yee Ho, Shi-Ping Zhang, Zhang-Jin Zhang, Eric TatChi Ziea, Vivian Taam Wong. 2012. Acupressure, reflexology, and auricular acupressure for insomnia: A systematic review of randomized controlled trials. Sleep Medicine 13, 971-984. [CrossRef]

9. Adrienne Juarascio, Norma G. Cuellar, Nalaka S. GooneratneAlternative Therapeutics for Sleep Disorders 126-139. [CrossRef]

10. Jaung-Geng Lin, Yi-Hung Chen. 2012. The Role of Acupuncture in Cancer Supportive Care. The American Journal of Chinese Medicine 40, 219-229. [CrossRef]

11. Gerhard Litscher, Guangyu Cheng, Weiping Cheng, Lu Wang, Qianqian Niu, Xiao Feng, Ingrid Gaischek, Haixue Kuang. 2012. Sino-European Transcontinental Basic and Clinical High-Tech Acupuncture Studies-Part 2: Acute Stimulation Effects on Heart Rate and Its Variability in Patients with Insomnia. Evidence-Based Complementary and Alternative Medicine 2012, 1-5. [CrossRef]

12. Courtney Lee, Dawn Wallerstedt, Alaine Duncan, Alexandra York, Michael Hollifield, Richard C. Niemtzow, Stephen M. Burns, Wayne B. Jonas. 2011. Design and Rationale of a Comparative Effectiveness Study to Evaluate Two Acupuncture Methods for the Treatment of Headaches Associated with Traumatic Brain Injury. Medical Acupuncture 23:4, 237-247. [Abstract] [Full Text HTML] [Full Text PDF] [Full Text PDF with Links]

13. Anita H. Hickey. 2011. Military Medical Acupuncture and CAM: Next Steps. Medical Acupuncture 23:4, 281-285. [Abstract] [Full Text HTML] [Full Text PDF] [Full Text PDF with Links]

14. Alaine D. Duncan, Janet M. Liechty, Cathy Miller, Gail Chinoy, Richard Ricciardi. 2011. Employee Use and Perceived Benefit of a Complementary and Alternative Medicine Wellness Clinic at a Major Military Hospital: Evaluation of a Pilot Program. The Journal of Alternative and Complementary Medicine 17:9, 809-815. [Abstract] [Full Text HTML] [Full Text PDF] [Full Text PDF with Links]

15. Edzard Ernst, Myeong Soo Lee, Tae-Young Choi. 2011. Acupuncture for insomnia? An overview of systematic reviews. European Journal of General Practice 17, 116-123. [CrossRef]

16. Yen-Ying Kung, Cheryl C.H. Yang, Jen-Hwey Chiu, Terry B.J. Kuo. 2011. The relationship of subjective sleep quality and cardiac autonomic nervous system in postmenopausal women with insomnia under auricular acupressure. Menopause 18, 638-645. [CrossRef]

17. Kelly Galvin, Madelaine BishopNeurological system 475-563. [CrossRef]

18. David O'Regan, Jacqueine Filshie. 2010. Acupuncture and cancer. Autonomic Neuroscience 157, 96-100. [CrossRef]

19. Jia-Ling Sun, Mei-Sheng Sung, Mei-Yu Huang, Guang-Chih Cheng, Chia-Chin Lin. 2010. Effectiveness of acupressure for residents of long-term care facilities with insomnia: A randomized controlled trial. International Journal of Nursing Studies 47, 798-805. [CrossRef] 
20. Gerrit J De Niet, Bea G Tiemens, Margot W Kloos, Giel JM Hutschemaekers. 2009. Review of systematic reviews about the efficacy of non-pharmacological interventions to improve sleep quality in insomnia. International Journal of Evidence-Based Healthcare 7:10.1111/jbr.2009.7.issue-4, 233-242. [CrossRef]

21. Wing-Fai Yeung, Ka-Fai Chung, Yau-Kwong Leung, Shi-Ping Zhang, Andrew C.K. Law. 2009. Traditional needle acupuncture treatment for insomnia: A systematic review of randomized controlled trials. Sleep Medicine 10, 694-704. [CrossRef]

22. T. I. Usichenko, Ch. Lehmann, E. Ernst. 2008. Auricular acupuncture for postoperative pain control: a systematic review of randomised clinical trials. Anaesthesia 63:10.1111/ana.2008.63.issue-12, 1343-1348. [CrossRef]

23. M. S. Lee, B.-C. Shin, L. K. P. Suen, T.-Y. Park, E. Ernst. 2008. Auricular acupuncture for insomnia: a systematic review. International Journal of Clinical Practice 62, 1744-1752. [CrossRef]

24. Melanie K. Means, Margaret D. Lineberger, Jack D. Edinger. 2008. Nonpharmacologic treatment of insomnia. Current Treatment Options in Neurology 10, 342-349. [CrossRef]

25. W LU, E DEANCLOWER, A DOHERTYGILMAN, D ROSENTHAL. 2008. The Value of Acupuncture in Cancer Care. Hematology/Oncology Clinics of North America 22, 631-648. [CrossRef]

26. Charlotte Paterson. 2008. The Colonization of the Lifeworld of Acupuncture: The SAR Conference. The Journal of Alternative and Complementary Medicine 14:2, 105-106. [Citation] [Full Text PDF] [Full Text PDF with Links]

27. Myeong Soo Lee, Byung-Cheul Shin. 2008. More About Auricular Acupuncture For Insomnia. The Journal of Alternative and Complementary Medicine 14:2, 106-108. [Citation] [Full Text PDF] [Full Text PDF with Links]

28. Xin-Yan Gao, Shi-Ping Zhang, Bing Zhu, Hong-Qi Zhang. 2008. Investigation of specificity of auricular acupuncture points in regulation of autonomic function in anesthetized rats. Autonomic Neuroscience 138, 50-56. [CrossRef]

29. M. Bijak. 2008. Fallbericht: Patientin mit Schlafstörung. Deutsche Zeitschrift für Akupunktur 51, 50-53. [CrossRef] 